\title{
An Efficient FPGA Implementation of Versatile Video Coding Intra Prediction
}

\author{
Hasan Azgin, Ercan Kalali, Ilker Hamzaoglu \\ Faculty of Engineering and Natural Sciences \\ Sabanci University \\ Istanbul, Turkey \\ \{hasanazgin, ercankalali, hamzaoglu\}@sabanciuniv.edu
}

\begin{abstract}
Versatile Video Coding (VVC) is a new international video compression standard offering much better compression efficiency than previous video compression standards at the expense of much higher computational complexity. In this paper, an efficient FPGA implementation of VVC intra prediction for angular prediction modes of $4 \times 4,8 \times 8,16 \times 16$ and $32 \times 32$ prediction unit sizes is proposed. In the proposed FPGA implementation, four constant multiplications used in one intra angular prediction equation are implemented using two DSP blocks and two adders in FPGA. The proposed FPGA implementation of VVC intra prediction, in the worst case, can process 34 full HD (1920x1080) frames per second.
\end{abstract}

Keywords - VVC, intra prediction, hardware implementation, FPGA, DSP block.

\section{INTRODUCTION}

ITU and ISO are developing a new international video compression standard called Versatile Video Coding (VVC) [1]-[6]. VVC will have higher compression efficiency than H.264 and High Efficiency Video Coding (HEVC) standards at the expense of much higher computational complexity [7]-[10].

Intra prediction algorithm predicts pixels of a block from the pixels of its already coded and reconstructed neighboring blocks. Intra prediction algorithm used in $\mathrm{VVC}$ is more complex than intra prediction algorithm used in HEVC. In HEVC, intra prediction unit (PU) size can be $4 \times 4,8 \times 8,16 \times 16$ and $32 \times 32$, for the luminance component of a video frame. There are 35 intra prediction modes for each PU size including DC and planar modes. 14848 intra prediction equations should be calculated for all PUs in a 32×32 coding unit (CU). HEVC intra angular prediction equations are 2-tap FIR filters [10].

In VVC, intra PU size can be $4 \times 4,8 \times 8,16 \times 16,32 \times 32$ and $64 \times 64$, for the luminance component of a video frame. There are 67 intra prediction modes for each PU size including DC and planar modes. 41288 intra prediction equations should be calculated for all PUs in a $32 \times 32$ CU. VVC intra angular prediction equations are either 4-tap gaussian or 4-tap cubic filters.

Xilinx FPGAs have built-in full-custom DSP blocks which can perform constant multiplications faster and with less energy than adders and shifters. A DSP block can be used to perform different constant multiplications by providing proper constant value to its input. Therefore, it is more efficient to implement constant multiplications using DSP blocks instead of using adders and shifters in an FPGA implementation.

In this paper, an efficient FPGA implementation of VVC intra prediction for angular prediction modes of $4 \times 4,8 \times 8$, $16 \times 16$ and $32 \times 32$ PU sizes is proposed. The proposed FPGA implementation uses 30 identical DSP datapaths (DDP). In the proposed FPGA implementation, intra angular prediction equations are manipulated in such a way that one intra angular prediction equation is implemented using two DSP blocks and two adders. Therefore, each DDP has two DSP blocks and two adders, and it can calculate any 4-tap gaussian and cubic filter used in VVC intra angular prediction in one clock cycle by changing DSP inputs.

The proposed VVC intra angular prediction hardware is implemented using Verilog HDL. The Verilog RTL code is verified to work at $119 \mathrm{MHz}$ on a Xilinx Virtex 7 FPGA. The proposed VVC intra angular prediction hardware, in the worst case, can process 34 full HD (1920x1080) frames per second.

Two VVC intra prediction hardware implementations are proposed in [11]. Several HEVC intra prediction hardware implementations are proposed in the literature [12]-[15]. In Section III, VVC intra prediction hardware proposed in this paper is compared with VVC and HEVC intra prediction hardware in the literature.

The rest of the paper is organized as follows. In Section II, VVC intra angular prediction algorithm is explained. In Section III, the proposed FPGA implementation of VVC intra angular prediction is presented, and its implementation results are given. Finally, Section IV presents the conclusions.

\section{VVC INTRA PREDICTION ALGORITHM}

VVC intra prediction algorithm predicts the pixels in prediction units (PU) of a coding unit (CU) using the pixels in the available neighboring PUs. For the luminance component of a frame, 4x4, 8x8, 16x16, 32x32 and 64x64 PU sizes are available. As shown in Fig. 1, there are 65 angular prediction modes (Mode) corresponding to different prediction angles (Angle) for each PU size. In addition, there are DC and planar prediction modes for each PU size. An 8x8 PU, four 4x4 PUs in it, and their neighboring pixels are shown in Fig. 2. 


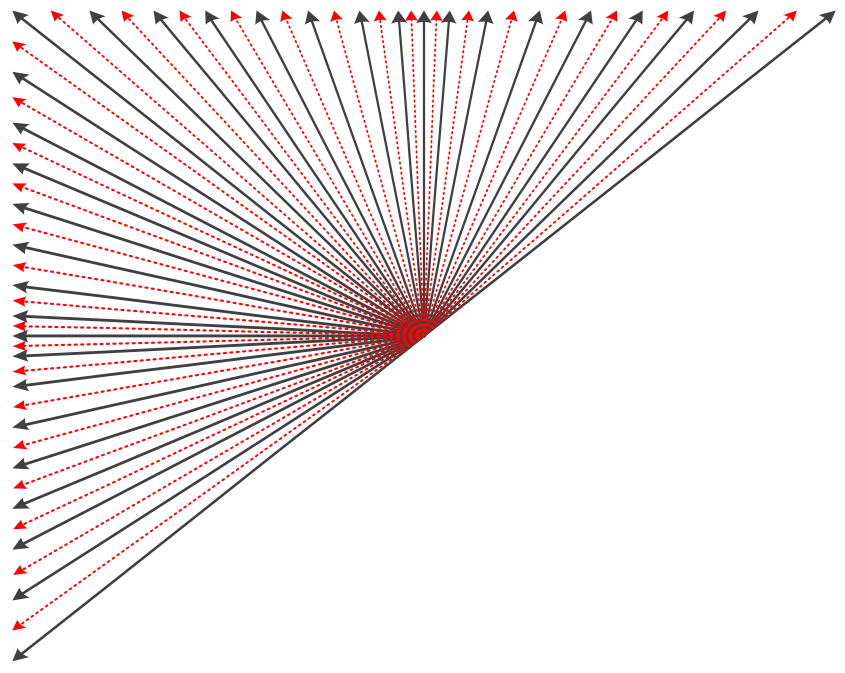

Fig. 1. VVC intra angular prediction modes.

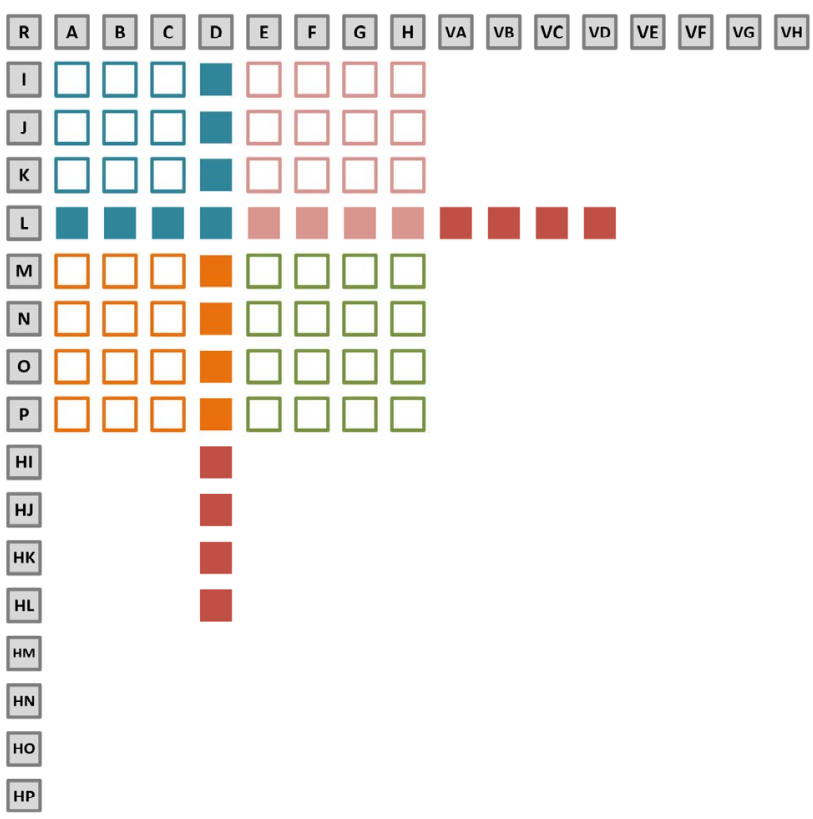

Fig. 2. Neighboring pixels of $4 \times 4$ and $8 \times 8$ PUs.

17 different 4-tap cubic filters and 17 different 4-tap gaussian filters are used as intra prediction equations. Cubic filters are used for $4 \times 4$ and $8 \times 8$ PUs. Gaussian filters are used for $16 \times 16$, $32 \times 32$ and $64 \times 64$ PUs.

In VVC intra prediction algorithm, first, reference main array is determined. Reference main array consists of pixels that will be used in intra prediction equations of the corresponding prediction mode and PU size. The reference main array is filled with above neighboring pixels if the prediction mode is equal to or greater than 34. However, if prediction angle is less than zero, first four pixels of the reference main array are filled with left neighboring pixels. The reference main array is filled with left neighboring pixels if the prediction mode is less than 34 . However, if the prediction angle is less than zero, first four pixels of the reference main array are filled with above neighboring pixels.
TABLE I. INTRA ANGULAR PREDICTION EQUATION REDUCTIONS BY DATA REUSE

\begin{tabular}{l|l|l|l|l|l|l}
\hline \hline & \multicolumn{3}{|c|}{ Cubic Filters } & \multicolumn{3}{c}{ Gaussian Filters } \\
\hline & $\begin{array}{l}\mathbf{4 x 4} \\
\text { PU }\end{array}$ & $\begin{array}{l}\text { 8x8 } \\
\text { PU }\end{array}$ & $\begin{array}{l}\mathbf{3 2 x 3 2} \\
\text { CU }\end{array}$ & $\begin{array}{l}\text { 16x16 } \\
\text { PU }\end{array}$ & $\begin{array}{l}\mathbf{3 2 x 3 2} \\
\text { PU }\end{array}$ & $\begin{array}{l}\mathbf{3 2 x 3 2} \\
\text { CU }\end{array}$ \\
\hline $\begin{array}{l}\text { \# of Pred. Equations } \\
\begin{array}{l}\text { \# of Pred. Equations } \\
\text { with Data Reuse }\end{array}\end{array}$ & 1040 & 4160 & 133120 & 16680 & 66560 & 133120 \\
\begin{tabular}{l} 
Reduction (\%) \\
\hline \hline
\end{tabular} & 61.06 & 74.95 & 77.85 & 84.43 & 90.02 & 91.13 \\
\hline
\end{tabular}

Then, deltaInt and deltaFract values are calculated as shown in (1a) and (1b), respectively. deltaInt is used to determine the positions of the pixels, that will be used in intra prediction equations, in reference main array. The four pixels that will be used in intra prediction equations are adjacent pixels in the reference main array, but they may not be adjacent in the video frame. These four pixels are selected as shown in (2a)-(2e), where $\mathrm{p}[0], \mathrm{p}[1], \mathrm{p}[2]$ and $\mathrm{p}[3]$ are the selected pixels from reference main array. If $\mathrm{p}[1]$ is the left-most pixel in the reference main array, $p[0]$ is equal to $p[1]$. If $p[2]$ is the rightmost pixel in the reference main array, $p[3]$ is equal to $p[2]$. PU size is used to determine whether cubic or gaussian filters will be used. deltaFract is used to determine which 4-tap filter among 17 4-tap filters will be used.

$$
\begin{gathered}
\text { deltaInt }=((y+1) * \text { Angle }) \gg 5 \\
\text { deltaFract }=((y+1) * \text { Angle }) \& 31 \\
\text { refMainIndex }=x+\text { deltaInt }+1 \\
p[1]=\text { refMain }[\text { refMainIndex }] \\
p[2]=\text { refMain }[\text { refMainIndex }+1] \\
p[0]=(x==0) ? p[1] \\
: \text { refMain }[\text { refMainIndex }-1] \\
p[3]=(x==(\text { width }-1)) ? p[2] \\
: \text { refMain }[\text { refMainIndex }+2] \\
x=0 \text { to }\left(P U_{\text {size }}-1\right), y=0 \text { to }\left(P U_{\text {size }}-1\right)
\end{gathered}
$$

\section{PROPOSED VVC INTRA PREDICTION HARDWARE}

In VVC, identical prediction equations are used in an intra angular prediction mode or in different intra angular prediction modes or in the intra angular prediction modes of different PU sizes. In the proposed hardware, data reuse technique is used to calculate identical prediction equations only once. There are 4x4 (PU size) x 65 (intra angular prediction modes) $=1040$ intra angular prediction equations for $4 \times 4$ PU size. Numbers of prediction equations for other PU sizes are shown in Table I. The number of prediction equations calculated for $4 \times 4$ PU size is reduced to 405 by using data reuse technique. Numbers of prediction equation reductions for other PU sizes are shown in Table I. 


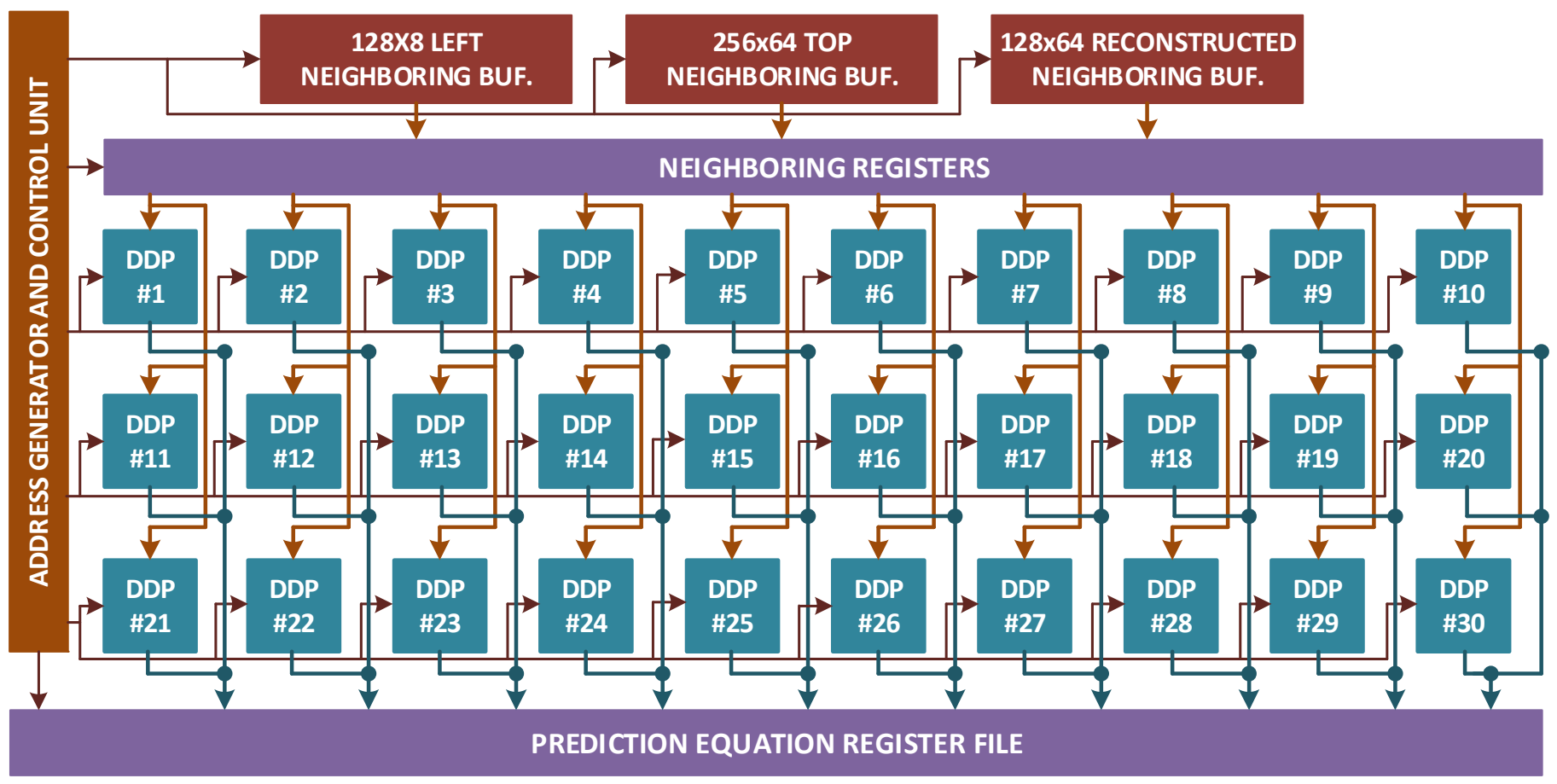

Fig. 3. Proposed VVC intra prediction hardware.

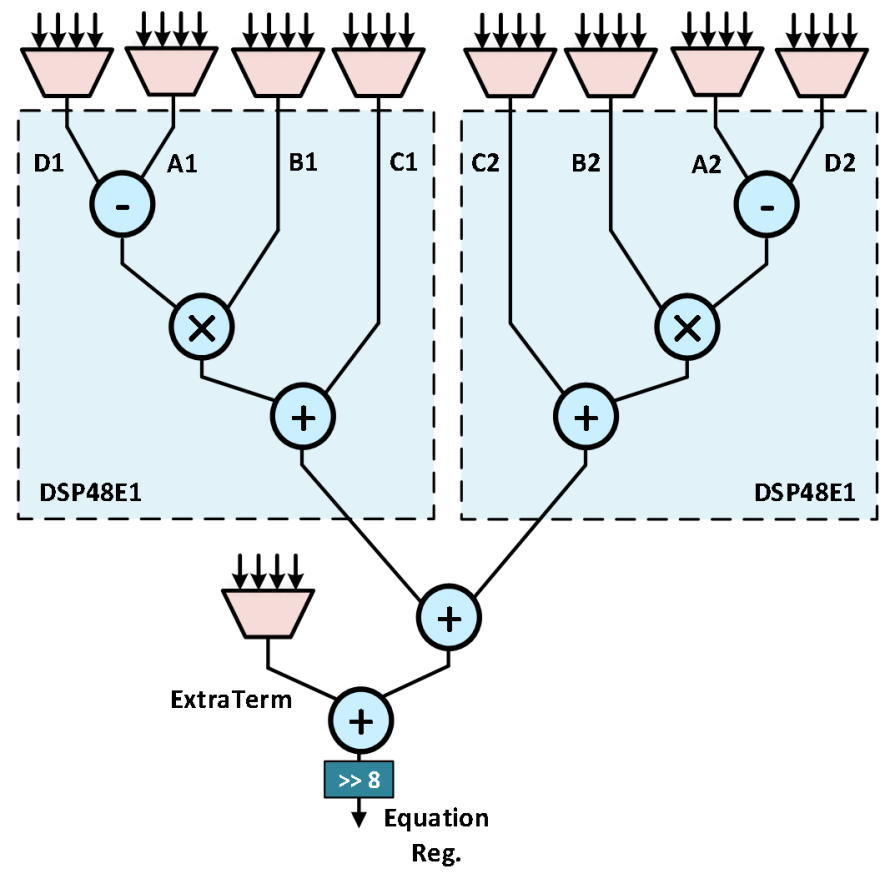

The proposed VVC intra prediction hardware is shown in Fig. 3. It implements 65 angular prediction modes for PU sizes from $4 \times 4$ to $32 \times 32$. It has thirty parallel reconfigurable DSP datapaths (DDP). One DDP, which can be configured to implement any of the 34 cubic and gaussian filters, is shown in Fig. 4.

$32 \times 32$ coding unit $(\mathrm{CU})$ is divided into $8 \times 8$ blocks and the neighboring pixels for the current $8 \times 8$ block and four $4 \times 4$ blocks within the current $8 \times 8$ block are loaded to registers. There are extra registers to store pixels from previous blocks, in case that an equation requires pixels from different $8 \times 8$ blocks. Therefore, the number of registers to store is decreased by storing only the neighboring pixels of $8 \times 8$ blocks, instead of keeping all neighboring pixels of $32 \times 32 \mathrm{CU}$.

FPGAs have built-in full-custom DSP blocks which can perform constant multiplications faster and with less energy than adders and shifters. A DSP block can be used to perform different constant multiplications by providing proper constant values to its inputs. Therefore, it is more efficient to implement constant multiplications using DSP blocks instead of using adders and shifters in an FPGA implementation.

Xilinx DSP block architecture is shown in Fig. 5. It has one pre-adder, one multiplier and one arithmetic logic unit (ALU). It also has optional pipeline registers. A DSP block can be configured to implement different operations.

In VVC, each intra angular prediction equation requires four multiplication operations to multiply four pixels with corresponding filter coefficients and three addition operations to add the results of these four multiplications. Therefore, four DSP blocks are necessary for implementing an intra angular prediction equation in its original form as in [11]. 


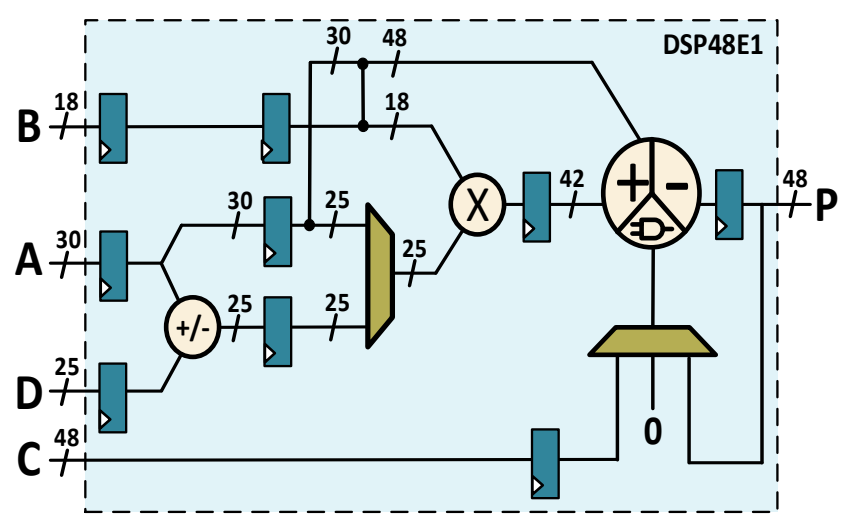

Fig. 5. Xilinx DSP48E1 block.

In the proposed FPGA implementation, intra angular prediction equations are manipulated in such a way that one intra angular prediction equation is implemented using two DSP blocks and two adders. Therefore, each DDP has two DSP blocks and two adders, and it can calculate any 4-tap gaussian and cubic filter used in VVC intra angular prediction in one clock cycle by changing A, B, C and D inputs of DSP blocks.

In the proposed FPGA implementation, DSP blocks are configured to implement equation (3). Each DDP implements equation (4).

$$
\begin{gathered}
P=B *(D-A)+C \\
P=(B 1 *(D 1-A 1)+C 1)+(B 2 *(D 2-A 2)+C 2) \\
+ \text { ExtraTerm }
\end{gathered}
$$

Four filter coefficients used in $34 \mathrm{VVC}$ intra angular prediction equations are shown in Table II. The A, B, C, D inputs of two DSP blocks and the extra term necessary for calculating each intra angular prediction equation using a DDP are also shown in Table II. The inputs of DSP blocks are shown in the order they appear in equation (4). Constant numbers are given to B inputs of two DSP blocks. Pixels or shifted pixels are given to D, A and C inputs of DSP blocks. Multiplexers are used to select the proper inputs for implementing each intra angular prediction equation.

For example, the intra angular prediction equation "Filter 1" shown in Table II is implemented using a DDP as shown in equations $(5 a),(5 b),(5 c)$.

$$
\begin{gathered}
P=(4 *(2 * p 3-p 2)+0)+(3 *(0-p 1)+(-p 4)) \\
+256 * p 2 \\
P=(8 * p 3-4 * p 2-3 * p 1-p 4+256 * p 2) \\
P=(-3 * p 1+252 * p 2+8 * p 3-p 4)
\end{gathered}
$$

The proposed VVC intra prediction hardware is implemented using Verilog HDL. The Verilog RTL code is synthesized, placed and routed to a Xilinx XC7VX485T FFG1157 FPGA with speed grade 3 using Xilinx Vivado
2017.2. The FPGA implementation is verified with post place and route simulations. The proposed FPGA implementation uses 5766 DFFs, 46382 LUTs, 4 BRAMs and 60 DSP48E1s blocks. It works at $119 \mathrm{MHz}$. It can process 34 full HD (1920x1080) video frames per second (fps).

The proposed VVC intra prediction hardware is compared with HEVC and VVC intra prediction hardware in the literature in Table III. Since VVC intra prediction algorithm is more complex than HEVC intra prediction algorithm, the proposed VVC intra prediction hardware implementation and the two VVC intra prediction hardware implementations proposed in [11] are slower and have more area than the HEVC intra prediction hardware implementations [12]-[15].

RECON_AS hardware implements VVC intra prediction using adders and shifters [11]. It does not use DSP blocks. RECON_DSP hardware implements VVC intra prediction using DSP blocks [11]. It uses four DSP blocks and one adder for implementing an intra angular prediction equation. The proposed VVC intra prediction hardware is faster than both RECON_AS and RECON_DSP hardware. It uses 50\% less DSP blocks than RECON_DSP hardware.

\section{CONCLUSION}

In this paper, an efficient FPGA implementation of VVC intra prediction for angular prediction modes of $4 \times 4,8 \times 8$, $16 \times 16$ and $32 \times 32$ PU sizes is proposed. The proposed reconfigurable datapath uses two DSP blocks and two adders to implement an intra angular prediction equation. The proposed VVC intra prediction hardware can process 34 full HD (1920x1080) frames per second on a Xilinx Virtex 7 FPGA.

\section{REFERENCES}

[1] J. Chen, Y. Chen, M. Karczewiz, X. Li, H. Liu, L. Zhang, X. Zhao, "Coding Tools Investigation for Next Generation Video Coding," ITU-T SG16 COM16-C806, Feb. 2015.

[2] J. Chen, E. Alshina, G. J. Sullivan, J. R. Ohm, J. Boyce, “Algorithm Description of Joint Exploration Model 7,” JVET-G1001, Jul. 2017.

[3] S. H. Park, E. S. Jang, "An Efficient Motion Estimation Method for QTBT Structure in JVET Future Video Coding," Data Compression Conference, Apr. 2017.

[4] M. J. Garrido, F. Pescador, M. Chavarrias, P. J. Lobo, and C. Sanz, "A high performance FPGA-based architecture for the future video coding adaptive multiple core transform," IEEE Trans. on Consumer Electronics, vol. 64, no. 1., pp. 53-60, Feb. 2018.

[5] A. C. Mert, E. Kalali, and I Hamzaoglu, "High Performance 2D Transform Hardware for Future Video Coding," IEEE Trans. on Consumer Electronics, vol. 63, no. 2, pp. 117-125, May 2017.

[6] H. Azgin, A. C. Mert, E. Kalali, and I. Hamzaoglu, "A reconfigurable fractional interpolation hardware for VCC motion compensation," Euromicro Conf. on Digital System Design (DSD), Aug. 2018.

[7] I. Hamzaoglu, O. Tasdizen, and E. Sahin, "An Efficient H.264 Intra Frame Coder System," IEEE Trans. on Consumer Electronics, vol. 54, no. 4, pp. 1903-1911, Nov. 2008.

[8] A. Abramowski and G. Pastuszak, "A novel intra prediction architecture for the hardware HEVC encoder," Euromicro Conf. on Digital System Design (DSD), Sep. 2013.

[9] P. Sjövall, J. Virtanen, J. Vanne, and T. D. Hamalainen, "High-level synthesis design flow for HEVC intra encoder on SoC-FPGA," Euromicro Conf. on Digital System Design (DSD), Aug. 2015. 
TABLE II. DDP CONFIGURATIONS

\begin{tabular}{|c|c|c|c|c|c|c|c|c|c|c|c|c|c|}
\hline \multirow{2}{*}{ Filters } & \multicolumn{4}{|c|}{ Filter Coefficients } & \multicolumn{4}{|c|}{ DSP Block 1} & \multicolumn{4}{|c|}{ DSP Block 2} & \multirow{2}{*}{ Extra Term } \\
\hline & Coeff1 & Coeff 2 & Coeff3 & Coeff 4 & B1 & D1 & A1 & $\mathrm{C1}$ & B2 & D2 & A2 & $\mathrm{C2}$ & \\
\hline 0 & 0 & 256 & 0 & 0 & 0 & 0 & $\mathrm{p} 2$ & 0 & 0 & 0 & 0 & 0 & $\mathrm{p} 2 \ll 8$ \\
\hline 1 & -3 & 252 & 8 & -1 & 4 & $\mathrm{p} 3 \ll 1$ & $\mathrm{p} 2$ & 0 & 3 & 0 & $\mathrm{p} 1$ & $(-\mathrm{p} 4)$ & $\mathrm{p} 2 \ll 8$ \\
\hline 2 & -5 & 247 & 17 & -3 & 9 & p3 & $\mathrm{p} 2$ & $\mathrm{p} 3 \ll 3$ & -3 & $\mathrm{p} 1$ & $(-p 4)$ & $(-\mathrm{p} 1) \ll 1$ & $\mathrm{p} 2 \ll 8$ \\
\hline 3 & -7 & 242 & 25 & -4 & 14 & 0 & $\mathrm{p} 2$ & $(-\mathrm{p} 4) \ll 2$ & 7 & $(-p 1)$ & $\mathrm{p} 3$ & $\mathrm{p} 3 \ll 5$ & $\mathrm{p} 2 \ll 8$ \\
\hline 4 & -9 & 236 & 34 & -5 & 5 & $(-\mathrm{p} 4)$ & $\mathrm{p} 2 \ll 2$ & 0 & 9 & $(-p 1)$ & $(-\mathrm{p} 3) \ll 2$ & $(-\mathrm{p} 3) \ll 1$ & $\mathrm{p} 2 \ll 8$ \\
\hline 5 & -10 & 230 & 43 & -7 & -43 & $(-\mathrm{p} 3)$ & $\mathrm{p} 2 \ll 1$ & $(-p 2) \ll 4$ & 10 & $\mathrm{p} 2 \ll 4$ & p1 & $(-\mathrm{p} 4) \ll 3$ & $\mathrm{p} 4$ \\
\hline 6 & -12 & 224 & 52 & -8 & 32 & p3 & $\mathrm{p} 2$ & $\mathrm{p} 1 \ll 3$ & 20 & p3 & $\mathrm{p} 1$ & $(-\mathrm{p} 4) \ll 3$ & $\mathrm{p} 2 \ll 8$ \\
\hline 7 & -13 & 217 & 61 & -9 & -9 & $\mathrm{p} 4$ & $\mathrm{p} 2$ & $(-p 3) \ll 2$ & 13 & $(-p 1)$ & $(-\mathrm{p} 2) \ll 4$ & $\mathrm{p} 3 \ll 66$ & p3 \\
\hline 8 & -14 & 210 & 70 & -10 & -12 & $\mathrm{p} 1$ & p2 & $(-\mathrm{p} 4) \ll 1$ & 70 & $\mathrm{p} 2$ & $-p 3$ & $(-p 4) \ll 3$ & $\mathrm{p} 2 \ll 7$ \\
\hline 9 & -15 & 203 & 79 & -11 & -75 & $(-\mathrm{p} 3)$ & p2 & $\mathrm{p} 3 \ll 2$ & 15 & $(-p 1)$ & $\mathrm{p} 4$ & $\mathrm{p} 4 \ll 2$ & $\mathrm{p} 2 \ll 7$ \\
\hline 10 & -16 & 195 & 89 & -12 & 61 & p3 & $\mathrm{p} 2$ & $(-\mathrm{p} 1) \ll 4$ & 12 & p3 & $\mathrm{p} 4$ & $\mathrm{p} 3 \ll<4$ & $\mathrm{p} 2 \ll 8$ \\
\hline 11 & -16 & 187 & 98 & -13 & 69 & p3 & $\mathrm{p} 2$ & $(-p 1) \ll 4$ & 13 & p3 & $\mathrm{p} 4$ & $\mathrm{p} 3 \ll 4$ & $\mathrm{p} 2 \ll 8$ \\
\hline 12 & -16 & 179 & 107 & -14 & -51 & $(-\mathrm{p} 3) \ll 1$ & $\mathrm{p} 2$ & $(-\mathrm{p} 1) \ll 4$ & 5 & p3 & $\mathrm{p} 4 \ll 1$ & $(-\mathrm{p} 4) \ll 2$ & $\mathrm{p} 2 \ll 7$ \\
\hline 13 & -16 & 170 & 116 & -14 & 86 & p3 & p2 & $(-\mathrm{p} 1) \ll 4$ & 14 & p3 & $\mathrm{p} 4$ & $\mathrm{p} 3 \ll<4$ & $\mathrm{p} 2 \ll 8$ \\
\hline 14 & -17 & 162 & 126 & -15 & -17 & p1 & $\mathrm{p} 2 \ll 1$ & $\mathrm{p} 4$ & 126 & p3 & 0 & $(-\mathrm{p} 4) \ll<4$ & $\mathrm{p} 2 \ll<7$ \\
\hline 15 & -16 & 153 & 135 & -16 & 103 & p3 & p2 & $(-p 1) \ll 4$ & 16 & p3 & $\mathrm{p} 4$ & $\mathrm{p} 3 \ll<4$ & $\mathrm{p} 2 \ll 8$ \\
\hline 16 & -16 & 144 & 144 & -16 & -144 & $(-\mathrm{p} 3)$ & p2 & 0 & 16 & $(-p 1)$ & $\mathrm{p} 4$ & 0 & 0 \\
\hline 17 & 47 & 161 & 47 & 1 & -161 & 0 & p2 & $\mathrm{p} 4$ & 47 & $\mathrm{p} 1$ & $(-p 3)$ & 0 & 0 \\
\hline 18 & 43 & 161 & 51 & 1 & -161 & 0 & $\mathrm{p} 2$ & $\mathrm{p} 4$ & 43 & $\mathrm{p} 1$ & $(-p 3)$ & $\mathrm{p} 3 \ll 3$ & 0 \\
\hline 19 & 40 & 160 & 54 & 2 & -32 & $(-p 1)$ & p2 & $\mathrm{p} 4 \ll 1$ & 54 & 0 & $(-p 3)$ & $\mathrm{p} 1 \ll 3$ & $\mathrm{p} 2 \ll 7$ \\
\hline 20 & 37 & 159 & 58 & 2 & -159 & 0 & $\mathrm{p} 2$ & $\mathrm{p} 4 \ll<1$ & 37 & $\mathrm{p} 1$ & $(-\mathrm{p} 3) \ll<1$ & $(-\mathrm{p} 3) \ll<4$ & 0 \\
\hline 21 & 34 & 158 & 62 & 2 & -62 & $(-\mathrm{p} 3)$ & $\mathrm{p} 2 \ll 1$ & 0 & 34 & $\mathrm{p} 1$ & $(-p 2)$ & $\mathrm{p} 4 \ll 1$ & 0 \\
\hline 22 & 31 & 156 & 67 & 2 & -5 & $(-\mathrm{p} 3)$ & $\mathrm{p} 2 \ll 2$ & $\mathrm{p} 2 \ll 3$ & 31 & $\mathrm{p} 1$ & $(-\mathrm{p} 3) \ll 1$ & $\mathrm{p} 4 \ll 1$ & $\mathrm{p} 2 \ll 7$ \\
\hline 23 & 28 & 154 & 71 & 3 & -71 & $(-\mathrm{p} 3)$ & $\mathrm{p} 2 \ll 1$ & $\mathrm{p} 1 \ll 5$ & 3 & $\mathrm{p} 4$ & $(-p 2) \ll 2$ & $(-\mathrm{p} 1) \ll 2$ & 0 \\
\hline 24 & 26 & 151 & 76 & 3 & -76 & $(-\mathrm{p} 3)$ & $\mathrm{p} 2 \ll 1$ & $(-\mathrm{p} 2)$ & 3 & $\mathrm{p} 1 \ll 3$ & $(-p 4)$ & $\mathrm{p} 1 \ll 1$ & 0 \\
\hline 25 & 23 & 149 & 80 & 4 & -21 & $(-p 1)$ & $\mathrm{p} 2$ & $\mathrm{p} 1 \ll 1$ & 80 & p3 & 0 & $\mathrm{p} 4 \ll 2$ & $\mathrm{p} 2 \ll 7$ \\
\hline 26 & 21 & 146 & 85 & 4 & -18 & 0 & p2 & $\mathrm{p} 4 \ll<2$ & 21 & $\mathrm{p} 1$ & $(-\mathrm{p} 3) \ll<2$ & p3 & $\mathrm{p} 2 \ll 7$ \\
\hline 27 & 19 & 142 & 90 & 5 & -14 & $(-\mathrm{p} 3)$ & $\mathrm{p} 2$ & $\mathrm{p} 4 \ll 2$ & 19 & $\mathrm{p} 1$ & $(-\mathrm{p} 3) \ll 2$ & $\mathrm{p} 4$ & $\mathrm{p} 2 \ll 7$ \\
\hline 28 & 17 & 139 & 94 & 6 & -11 & $(-p 3) \ll 3$ & $\mathrm{p} 2$ & $\mathrm{p} 1 \ll 4$ & 6 & p3 & $(-p 4)$ & $\mathrm{p} 1$ & $\mathrm{p} 2 \ll 7$ \\
\hline 29 & 16 & 135 & 99 & 6 & -7 & $(-p 3) \ll 4$ & p2 & $\mathrm{p} 1 \ll 4$ & 6 & $\mathrm{p} 4$ & $\mathrm{p} 3 \ll 1$ & $(-\mathrm{p} 3)$ & $\mathrm{p} 2 \ll 7$ \\
\hline 30 & 14 & 131 & 104 & 7 & -3 & $(-\mathrm{p} 3)<5$ & $\mathrm{p} 2$ & $\mathrm{p} 3 \ll 3$ & 7 & $\mathrm{p} 1 \ll 1$ & $(-p 4)$ & 0 & $\mathrm{p} 2 \ll 7$ \\
\hline 31 & 13 & 127 & 108 & 8 & -127 & 0 & p2 & $\mathrm{p} 4 \ll 3$ & 13 & $\mathrm{p} 1$ & $(-\mathrm{p} 3) \ll 3$ & $\mathrm{p} 3 \ll 2$ & 0 \\
\hline 32 & 11 & 123 & 113 & 9 & -113 & $(-\mathrm{p} 3)$ & $\mathrm{p} 2$ & $\mathrm{p} 1$ & 10 & $\mathrm{p} 1$ & $(-p 2)$ & $\mathrm{p} 4 \ll 3$ & $\mathrm{p} 4$ \\
\hline 33 & 10 & 118 & 118 & 10 & -118 & $(-\mathrm{p} 3)$ & p2 & 0 & 10 & $\mathrm{p} 1$ & $(-p 4)$ & 0 & 0 \\
\hline
\end{tabular}

TABLE III. HARDWARE COMPARISON

\begin{tabular}{|c|c|c|c|c|c|c|c|c|}
\hline & {$[10]$} & [12] & [13] & [14] & [15] & $\begin{array}{c}{[11]} \\
\text { RECON AS }\end{array}$ & $\begin{array}{c}{[11]} \\
\text { RECON_DSP }\end{array}$ & Proposed \\
\hline FPGA & Xilinx 6 & Stratix III & Arria II GX & Virtex 6 & Xilinx 6 & Virtex 7 & Virtex 7 & Virtex 7 \\
\hline FPGA Technology & $40 \mathrm{~nm}$ & $65 \mathrm{~nm}$ & $40 \mathrm{~nm}$ & $40 \mathrm{~nm}$ & $40 \mathrm{~nm}$ & $28 \mathrm{~nm}$ & $28 \mathrm{~nm}$ & $28 \mathrm{~nm}$ \\
\hline Standard & HEVC & HEVC & HEVC & HEVC & HEVC & VVC & VVC & VVC \\
\hline DFF & 849 & $5.5 \mathrm{~K}$ & $110 \mathrm{~K}$ & --- & 2006 & 6234 & 4076 & 5766 \\
\hline LUT & 2381 & $14 \mathrm{~K}$ & $170 \mathrm{~K}$ & $24 \mathrm{~K}$ & 6013 & 49556 & 32499 & 46382 \\
\hline BRAM & $3.2 \mathrm{~KB}$ & $6 \mathrm{~KB}$ & -- & $6 \mathrm{~KB}$ & $3.2 \mathrm{~KB}$ & $3.2 \mathrm{~KB}$ & $3.2 \mathrm{~KB}$ & $3.2 \mathrm{~KB}$ \\
\hline DSP Block & --- & --- & --- & --- & --- & -- & 120 & 60 \\
\hline Max Freq. (MHz) & 150 & 110 & 219 & 100 & 166 & 108 & 105 & 119 \\
\hline Frames per Sec. & $\begin{array}{c}30 \\
1920 \times 1080\end{array}$ & $\begin{array}{c}30 \\
3840 \times 2160\end{array}$ & $\begin{array}{c}24 \\
3840 \times 2160\end{array}$ & $\begin{array}{c}60 \\
1920 \times 1080\end{array}$ & $\begin{array}{c}40 \\
1920 \times 1080\end{array}$ & $\begin{array}{c}30 \\
1920 \times 1080\end{array}$ & $\begin{array}{c}30 \\
1920 \times 1080\end{array}$ & $\begin{array}{c}34 \\
1920 \times 1080\end{array}$ \\
\hline PU Size & 4,8 & $4,8,16,32$ & $4,8,16,32$ & $4,8,16,32$ & $4,8,16,32$ & $4,8,16,32$ & $4,8,16,32$ & $4,8,16,32$ \\
\hline
\end{tabular}


[10] E. Kalali, Y. Adibelli, and I. Hamzaoglu, "A High Performance and Low Energy Intra Prediction Hardware for High Efficiency Video Coding," Int. Conf. on Field Programmable Logic and Applications, pp. 719-722, Aug. 2012.

[11] H. Azgin, A. C. Mert, E. Kalali, I. Hamzaoglu, "Reconfigurable Intra Prediction Hardware for Future Video Coding," IEEE Trans. on Consumer Electronics, vol. 63, no. 4, pp. 419-425, Nov. 2017.

[12] B. Min, Z. Xu, R. C. C. Cheung, "A Fully Pipelined Hardware Architecture for Intra Prediction of HEVC," IEEE Trans. on Circuits and Systems for Video Technology, Jul. 2016.

[13] F. Amish, E. B. Bourennane, "Fully Pipelined Real Time Hardware Solution for High Efficiency Video Coding (HEVC) Intra Prediction," Journal of System Architecture, vol. 64, pp. 133-147, Mar. 2016.

[14] G. Pastuszak, A. Abramowski, "Algorithm and Architecture Design of The H.265/HEVC Intra Encoder," IEEE Trans. on Circuits and Systems for Video Technology, vol. 26, no. 1, pp. 210-222, Jan. 2016.

[15] H. Azgin, E. Kalali, I. Hamzaoglu, "A Computation and Energy Reduction Technique for HEVC Intra Prediction," IEEE Trans. on Consumer Electronics, vol. 63, no. 1, pp. 36-43, Feb. 2017. 